\title{
SITUAÇÃO DA PESQUISA EM ENFERMAGEM EM HOSPITAIS DO MUNICÍPIO DE SÃO PAULO*
}

\author{
Nilce Piva Adami** \\ Sonia Della Torre Salzano*** \\ Rosa Apparecida Pimenta de Castro**** \\ Maguida Costa Stefanelli ${ }^{\star \star \star \star \star}$
}

ADAMI, N. P.; SALZANO, S.D.T.; CASTRO, R.A.P. de; STEFANELLI, M.C. Situação da pesquisa em enfermagem em hospitais do município de São Paulo. Rev.latinoam.enfermagem, Ribeirão Preto, v. 4, n. 1, p. 5-20, janeiro 1996.

Tendo por objetivo descrever a situação da pesquisa em enfermagem em hospitais do município de São Paulo, no período de 1989 a 1993, foi realizada uma pesquisa exploratória, descritiva, abrangendo 14 estabelecimentos sendo sete públicos e sete da rede privada. Nos públicos foram produzidas 114 pesquisas e nos privados 40, predominando as apresentadas em eventos científicos. Foram expressas as facilidades e dificuldades para a produção de pesquisa nestas instituições, bem como, a incorporação dos resultados destas na prática, principalmente quando o objetivo de estudo emerge de problemas percebidos no processo assistencial. Centros de pesquisa, parceria serviço-universidade e contratação de enfermeiras pesquisadoras foram estratégias adotadas para o desenvolvimento de pesquisas em algumas das instituições estudadas.

UNITERMOS: pesquisa, pesquisa em enfermagem, produção científica

\footnotetext{
* Trabalho apresentado no 70 SENPE, junho de 1994, Fortaleza-CE, Brasil

** Professor Livre-Docente do Departamento de Enfermagem da Universidade Federal de São Paulo

*** Professor Titular da Escola de Enfermagem da Universidade de São Paulo

**** Professor Adjunto do Departamento de Enfermagem da Universidade Federal de São Paulo

***** Professor Titular pela Escola de Enfermagem da Universidade de São Paulo e Professor Visitante da Universidade Federal do Paraná
} 


\section{INTRODUÇÃO}

A pesquisa está sendo cada vez mais ressaltada, nas falas das enfermeiras e na literatura específica, como o caminho em busca da qualidade da assistência de enfermagem, preocupação central do exercício profissional.

Parte da essência do problema da pesquisa em enfermagem está na sua utilização, não importando se a pesquisa foi construída com base em conhecimentos filosóficos e teóricos, por quem domina o conhecimento em centros de pesquisa ou programas de pós-graduação ou se o estudo emergiu de um problema percebido no dia-a-dia das atividades profissionais. O importante é adequá-la e decodificá-la para implementação na prática da enfermagem para assegurar sua qualidade, o que deve ser feito junto as enfermeiras envolvidas diretamente com a assistência de nossa clientela.

Segundo MOLONEY (1986) é importante que as enfermeiras assistenciais e pesquisadoras unam seus esforços em prol do reconhecimento da profissão e da qualidade de excelência da enfermagem, realizando pesquisas em conjunto.

Necessário se faz destacar que contamos, no País, com mais de 100 cursos de graduação em enfermagem oferecendo programas de iniciação científica, o que também, é realizado por algumas seções da Associação Brasileira de Enfermagem (ABEn). Os programas de pós-graduação, sem exceção, oferecem cursos avançados de pesquisa, orientam a execução das pesquisas dos alunos, que em geral voltam às suas escolas de origem para exercerem a docência ou para instituições onde se dedicam às atividades assistenciais. Estes programas já titularam aproximadamente 750 mestres e 65 doutores (STEFANELLI; FRIEDLANDER, 1992); em 1994, contamos com cerca de 800 mestres e 100 doutores.

A produção científica da enfermagem brasileira, resultante destes programas é substancial, marcadamente na década de 80 , época em que se somam a produção de mestres, doutores e de mestrandos e doutorandos, oriundos dos cursos de mestrado e doutorado, criados respectivamente a partir de 1971 e 1981.

CIANCIARULLO; SALZANO (1991) realizaram um estudo comparativo, a partir dos achados de NOGUEIRA (1982) acrescidos dos dados da década de 80, sobre a produção científica das enfermeiras, segundo áreas de investigação onde foi possível constatar que $98 \%$ dessa produção coincide com os 20 anos de pós-graduação em enfermagem no País.

Embora o título de mestre ou doutor, em geral, não seja valorizado pelas instituições de saúde, parece estar havendo uma mudança nesta tendência, pois os programas de mestrado e doutorado contam com um crescente aumento de enfermeiras da área assistencial (STEFANELLI; FRIEDLANDER, 1992).

Tendo em vista estes esforços das escolas em nível de graduação e pósgraduação e de seções da ABEn, sabemos que existe ainda um hiato entre a pesquisa e a prática profissional que precisa ser suprido, problema este, também sentido em âmbito internacional. (CHASKA, 1983; HORSLEY et al, 1983; JOLLEY; ALLAN, 1989; PHILLIPS, 1986).

A inquietação sobre a distância existente entre enfermeiras pesquisadoras e assistenciais e como estas estão desenvolvendo e utilizando os resultados das 
investigações levou-nos a realizar o presente estudo, que tem como objetivo retratar a situação da pesquisa no âmbito da enfermagem em alguns hospitais do município de São Paulo.

\section{METODOLOGIA}

O método utilizado foi o exploratório descritivo e a coleta de dados foi realizada em sete hospitais da rede privada e sete da rede pública, sendo todos eles campo de estágio para alunos de enfermagem. Forneceram os dados para este estudo as enfermeiras que responderam o questionário até abril de 1994, sobre as pesquisas realizadas nos últimos cinco anos - 1989 a 1993 inclusive.

O questionário utilizado para a coleta de dados contém perguntas fechadas e abertas; as primeiras foram utilizadas para caracterizar a instituição e o quadro de pessoal de enfermagem e as demais para obter dados sobre o objeto do estudo. $O$ instrumento foi testado com três enfermeiras assistenciais tendo sofrido algumas modificações para melhor compreensão das questões.

Os questionários, acompanhados da carta justificando a finalidade do estudo (Anexo I), foram entregues diretamente às enfermeiras responsáveis pelos serviços de enfermagem das instituições campo de estudo, que os devolveram devidamente preenchidos. $O$ anonimato foi respeitado em relação às instituições e às respondentes.

\section{APRESENTAÇÃO E DISCUSSÃO DE RESULTADOS}

\section{Caracterização dos hospitais}

A caracterização dos hospitais estudados pode ser visualizada nas Tabelas 1 e 2, apresentadas a seguir:

TABELA 1 - HOSPITAIS ESTUDADOS SEGUNDO CLASSIFICAÇÃO E TIPO DE
ESTABELECIMENTO - MUNICÍPIO DE SÃO PAULO, ABRIL DE 1994

\begin{tabular}{|c|c|c|c|c|}
\hline \multirow{3}{*}{ CLASSIFICAÇÃO } & \multicolumn{2}{|c|}{ TIPO } & \multirow{2}{*}{\multicolumn{2}{|c|}{ TOTAL }} \\
\hline & \multirow{2}{*}{$\begin{array}{c}\text { PÚBLICO } \\
\mathrm{N}^{\mathrm{O}}\end{array}$} & \multirow{2}{*}{$\begin{array}{c}\text { PRIVADO } \\
\text { No }^{\circ} \\
\end{array}$} & & \\
\hline & & & $\mathrm{N}^{\mathrm{O}}$ & $\%$ \\
\hline Geral & 3 & 7 & 10 & 71,4 \\
\hline Especializado & 4 & - & 4 & 28,6 \\
\hline TOTAL & 7 & 7 & 14 & 100,0 \\
\hline
\end{tabular}


Os dados constantes na Tabela 1 mostram que dos 14 hospitais estudados, sete pertencem ao setor público $(50,0 \%)$ e sete à rede privada $(50,0 \%)$, dos quais dez são gerais $(71,4 \%)$ e quatro especializados $(28,6 \%)$.

TABELA 2 - HOSPITAIS ESTUDADOS SEGUNDO FINALIDADE E TIPO DO ESTABELECIMENTO - MUNICÍPIO DE SÃO PAULO, ABRIL DE 1994

\begin{tabular}{lccccc}
\hline & \multicolumn{2}{c}{ TIPO } & \multicolumn{2}{c}{ TOTAL } \\
\cline { 2 - 4 } CLASSIFICAÇÃO & PÚBLICO & PRIVADO & & $N^{\circ}$ & $\%$ \\
\hline Assistência & $N^{\circ}$ & $N^{\circ}$ & & 4 & 28,6 \\
Assistência e & - & 1 & 1 & 7,1 \\
$\begin{array}{l}\text { ensino } \\
\text { Assistência, ensino }\end{array}$ & - & & & 64,3 \\
e pesquisa & 7 & 2 & 9 & 100,0 \\
\hline TOTAL & 7 & 7 & 14 & 14 \\
\hline
\end{tabular}

No referente à finalidade, quatro são assistenciais (28,6\%), um é assistencial e de ensino $(7,1 \%)$ e nove $(64,3 \%)$ incluem dentre suas finalidades, a assistência, 0 ensino e a pesquisa, nos quais estão inseridos todos os hospitais públicos estudados (Tabela 2).

Quanto ao número de leitos disponíveis em atividade nos hospitais estudados, os dados da Tabela 3 mostram que a maioria é de grande porte, segundo classificação adotada pelo Ministério da Saúde, 1983.

TABELA 3 - HOSPITAIS ESTUDADOS SEGUNDO NÚMERO DE LEITOS, NÚMERO E PORCENTAGEM DE PESSOAL DE ENFERMAGEM, RELAÇÃO ENFERMEIRA E PESSOAL DE ENFERMAGEM POR 10 LEITOS - MUNICÍPIO DE SÃO PAULO, ABRIL DE 1994.

\begin{tabular}{|c|c|c|c|c|c|c|c|c|}
\hline \multirow[b]{2}{*}{ Hospitais } & \multirow[b]{2}{*}{$\begin{array}{l}\text { N. }{ }^{\circ} \text { de } \\
\text { leitos }\end{array}$} & \multirow[b]{2}{*}{$\begin{array}{c}\mathrm{n} .^{\circ} \mathrm{de} \\
\text { pess.enf. }\end{array}$} & \multicolumn{4}{|c|}{$\%$ de pess.de enf. } & \multicolumn{2}{|c|}{ Relação } \\
\hline & & & $E^{1}$ & $\mathrm{TE}^{2}$ & $A E^{3}$ & $\mathrm{AT}^{4}$ & $\begin{array}{l}\text { E/por } 10 \\
\text { leitos }\end{array}$ & $\begin{array}{c}\text { Pess. Enf./ por } \\
10 \text { leitos }\end{array}$ \\
\hline \multicolumn{9}{|l|}{ Públicos } \\
\hline A1 & 219 & 310 & 27,1 & - & 69,7 & 3,2 & 3,8 & 14,1 \\
\hline B1 & 620 & 1720 & 15,2 & 5,6 & 46,6 & 32,6 & 4,2 & 27,7 \\
\hline C1 & 305 & 631 & 24,1 & 9,0 & 57,4 & 9,5 & 4,9 & 20,6 \\
\hline D1 & 272 & 641 & 27,4 & 6,2 & 31,8 & 34,6 & 6,4 & 23,6 \\
\hline E1 & 801 & 1604 & 16,8 & - & 57,9 & 25,3 & 3,3 & 20,0 \\
\hline F1 & 280 & 273 & 14,3 & - & 52,0 & 33,7 & 1,3 & 9,7 \\
\hline G1 & 150 & 420 & 19,5 & - & 25,7 & 54,8 & 5,4 & 28,0 \\
\hline \multicolumn{9}{|l|}{ Privados } \\
\hline $\mathrm{A} 2$ & 170 & 450 & 20,0 & - & 65,5 & 14,5 & 5,2 & 26,4 \\
\hline B2 & 1300 & 1759 & 13,6 & 0,7 & 45,4 & 40,3 & 1,8 & 13,5 \\
\hline $\mathrm{C} 2$ & 146 & 331 & 42,3 & 22,0 & 24,5 & 11,2 & 9,5 & 22,7 \\
\hline D2 & 196 & 367 & 22,4 & 7,3 & 66,8 & 3,5 & 4,1 & 18,7 \\
\hline E2 & 203 & 525 & 27,0 & 5,7 & 60,6 & 6,7 & 6,9 & 25,8 \\
\hline $\mathrm{F} 2$ & 800 & 1730 & 12,8 & - & 46,2 & 41,0 & 2,7 & 21,6 \\
\hline G2 & 337 & 793 & 20,3 & - & 57,0 & 22,7 & 4,7 & 23,5 \\
\hline 1. Enferme & & & ni & nfe & $m$ & & & \\
\hline 3. Auxiliar & nferm & & end & e er & agem & & & \\
\hline
\end{tabular}


Quanto ao número de pessoal de enfermagem empregado nessas instituições, observa-se que entre os hospitais públicos os denominados $D_{1}$ e $A_{1}$ foram os que apresentaram maiores percentuais de enfermeiras, $27,4 \%$ e $27,1 \%$, respectivamente. Quanto à categoria de auxiliar de enfermagem os maiores percentuais foram identificados nos Hospitais $A_{1}(69,7 \%)$ e $E_{1}(57,9 \%)$, e quanto aos atendentes de enfermagem, os maiores valores foram nos hospitais $G_{1}(54,8 \%)$ e $D_{1}(34,6 \%)$. A categoria do técnico de enfermagem estava representada apenas em três hospitais.

Com referência ao percentual do pessoal de enfermagem nos hospitais privados, destacou-se o $\mathrm{C}_{2}$ que possuía $42,3 \%$ de enfermeiras representando quase que o dobro dos valores encontrados nos Estabelecimentos $E_{2}$ e $D_{2}$. Quanto ao percentual de auxiliares de enfermagem, destacaram-se os Estabelecimentos $D_{2}$ $(66,8 \%), A_{2}(65,5 \%)$ e $E_{2}(60,6 \%)$. Em relação ao percentual de atendentes de enfermagem, os maiores valores foram encontrados nos Estabelecimentos $F_{2}(41,0 \%)$ e $\mathrm{B}_{2}(40,3 \%)$.

Pode-se perceber ainda, que para cada dez leitos há uma ligeira predominância no quantitativo de enfermeiras nas instituições privadas.

Comparando-se os resultados encontrados com os da pesquisa realizada em 1983 pelo COFEN/ABEn (1985), referentes ao Estado de São Paulo, observa-se que eles não se enquadram na média estadual, excetuando-se o percentual da categoria do técnico de enfermagem que permaneceu estável nos hospitais públicos $(3,5 \%)$ e diminuiu nos privados $(2,4 \%)$. Destaca-se ainda, a redução acentuada dos atendentes de enfermagem e o aumento dos auxiliares de enfermagem e enfermeiras, o que leva a supor que os hospitais estudados estão qualificando os atendentes e deixando de contratar novos por força da aplicação da legislação que dispõe e regulamenta 0 exercício profissional de enfermagem.

\section{A produção de pesquisas nos últimos cinco anos}

TABELA 4 - HOSPITAIS ESTUDADOS POR TIPO DE ESTABELECIMENTO, SEGUNDO A REALIZAÇÃO OU NÃO DE PESQUISAS E 0 DESENVOLVIMENTO DESTAS SÓ POR ENFERMEIRAS E POR ENFERMEIRAS EM GRUPO MULTIPROFISSIONAL - MUNICÍPIO DE SÃO PAULO, ABRIL DE 1994

\begin{tabular}{ccccc}
\hline & \multicolumn{4}{c}{ Pesquisas Realizadas } \\
\cline { 2 - 5 } $\begin{array}{c}\text { TIPO DE } \\
\text { ESTABELECIMENTO }\end{array}$ & SIM & NÃO & $\begin{array}{c}\text { SÓ POR } \\
\text { ENFERMEIRAS }\end{array}$ & $\begin{array}{c}\text { ENFERMEIRAS EM GRUPO } \\
\text { MULTIPROFISSIONAL }\end{array}$ \\
\hline Público & 7 & - & 7 & 6 \\
Privado & 5 & 2 & 5 & 4 \\
\hline TOTAL & 12 & 2 & 12 & 10 \\
\hline
\end{tabular}


Pelos dados da Tabela 4 observa-se que dos 14 hospitais estudados, 12 informaram a realização de pesquisas no período de 1989 a $1993(85,7 \%)$ nos quais estão incluídos todos os estabelecimentos públicos. Observa-se que há trabalhos realizados só por enfermeiras nos 12 hospitais estudados, bem como a participação delas em estudos multiprofissionais, em seis estabelecimentos públicos e quatro privados.

TABELA 5 - PESQUISAS REALIZADAS EM HOSPITAIS PÚBLICOS POR ENFERMEIRAS E POR ENFERMEIRAS EM GRUPO MULTIPROFISSIONAL NO PERÍODO DE 1989 A 1993, SEGUNDO NÚMERO DE AUTORES, PUBLICAÇÃO E APRESENTAÇÃO EM EVENTOS CIENTÍFICOS - MUNICÍPIO DE SÃO PAULO, ABRIL DE 1994

\begin{tabular}{|c|c|c|c|c|c|c|c|c|c|c|}
\hline \multirow{3}{*}{$\begin{array}{l}\text { Hospitais } \\
\text { Públicos }\end{array}$} & \multirow[b]{3}{*}{$\begin{array}{l}N{ }^{\circ} \text { de } \\
\text { Pesq. }\end{array}$} & \multirow[b]{3}{*}{$\begin{array}{c}\bar{X} \\
\text { Ano }\end{array}$} & & & & & \multicolumn{4}{|c|}{ Pesquisas Realizadas } \\
\hline & & & \multicolumn{4}{|c|}{ N. ${ }^{\circ}$ de autores } & \multicolumn{2}{|c|}{ Publicação } & \multicolumn{2}{|c|}{ Apresentação } \\
\hline & & & 1 & 2 & 3 & $4 e+$ & $\operatorname{sim}$ & não & $\operatorname{sim}$ & não \\
\hline A1 & 44 & 8,8 & 3 & 12 & 10 & 19 & 6 & 38 & 33 & 11 \\
\hline B1 & 22 & 4,4 & 4 & 8 & 2 & 8 & 13 & 9 & 20 & 2 \\
\hline $\mathrm{C} 1$ & 22 & 4,4 & 1 & 3 & 5 & 13 & 12 & 10 & 17 & 5 \\
\hline D1 & 12 & 2,4 & 3 & 2 & 3 & 4 & 8 & 4 & 10 & 2 \\
\hline E1 & 10 & 2,0 & 2 & 4 & 3 & 1 & 2 & 8 & 10 & - \\
\hline F1 & 3 & 0,6 & - & 1 & 2 & - & 2 & 1 & 3 & - \\
\hline G1 & 1 & 0,2 & - & - & - & 1 & - & 1 & 1 & - \\
\hline TOTAL & 114 & 22,8 & 13 & 30 & 25 & 46 & 43 & 71 & 94 & 20 \\
\hline
\end{tabular}

Em relação aos hospitais públicos, os dados da Tabela 5 evidenciam que no período considerado foram produzidas 114 pesquisas com média anual de 22,8 , sendo que dessas 43 foram publicadas e 94 apresentadas em eventos científicos.

No referente à titulação dos autores os dados indicam maior número de enfermeiras com especialização.

TABELA 6 - PESQUISAS REALIZADAS EM HOSPITAIS PRIVADOS POR ENFERMEIRAS E POR ENFERMEIRAS EM GRUPO MULTIPROFISSIONAL, NO PERÍODO DE 1989 A 1993, SEGUNDO NÚMERO DE AUTORES, PUBLICAÇÃO E APRESENTAÇÃO EM EVENTOS CIENTÍFICOS - MUNICÍPIO DE SÃO PAULO, ABRIL DE 1994

\begin{tabular}{|c|c|c|c|c|c|c|c|c|c|c|}
\hline \multirow{3}{*}{$\begin{array}{l}\text { Hospitais } \\
\text { Públicos* }\end{array}$} & \multirow[b]{3}{*}{$\begin{array}{l}\text { N. }{ }^{\circ} \text { de } \\
\text { Pesq. }\end{array}$} & \multirow[b]{3}{*}{$\begin{array}{c}X \\
\text { Ano } \\
\end{array}$} & & & & & \multicolumn{4}{|c|}{ Pesquisas Realizadas } \\
\hline & & & \multicolumn{4}{|c|}{ N. ${ }^{\circ}$ de autores } & \multicolumn{2}{|c|}{ Publicação } & \multicolumn{2}{|c|}{ Apresentação } \\
\hline & & & 1 & 2 & 3 & $4 e+$ & $\operatorname{sim}$ & não & $\operatorname{sim}$ & não \\
\hline $\mathrm{A} 2$ & 13 & 2,6 & - & 5 & 2 & 6 & 6 & 7 & 13 & - \\
\hline B2 & 11 & 2,2 & 1 & 1 & 5 & 4 & 5 & 6 & 6 & 5 \\
\hline $\mathrm{C} 2$ & 7 & 1,4 & 2 & 1 & 2 & 2 & 3 & 4 & 5 & 2 \\
\hline D2 & 5 & 1,0 & 1 & 1 & 1 & 2 & - & 5 & 5 & - \\
\hline E2 & 4 & 0,8 & - & 1 & 1 & 2 & 3 & 1 & 1 & 3 \\
\hline TOTAL & 40 & 8,0 & 4 & 9 & 11 & 16 & 17 & 23 & 30 & 10 \\
\hline
\end{tabular}

*dois hospitais privados informaram que as enfermeiras não realizam pesquisas nos últimos cinco anos 
Pelos dados da Tabela 6 pode-se observar que foram realizadas 40 pesquisas nos anos de 1989 a 1993, com a média anual de 8,0. Destas 40 pesquisas 17 foram publicadas. Em um dos hospitais privados o percentual de enfermeiras foi bem maior $(27,0 \%)$, e, no entanto apresentou o menor número de pesquisas produzidas (Tabelas 3 e 6), isto sem considerar outros fatores.

Em relação à qualificação dos autores, prevaleceu também, em geral, as enfermeiras com especialização.

O confronto dos dados das Tabelas 5 e 6 mostra claramente que as enfermeiras dos hospitais públicos estudados desenvolveram e participaram de maior número de pesquisas, quase três vezes mais do que as enfermeiras do estabelecimentos privados, sendo que destes somente dois tinham por finalidade a investigação. As respondentes de todos os hospitais públicos assinalaram que os mesmos tinham por finalidade a assistência, o ensino e a pesquisa.

Dos 12 hospitais cujos serviços de enfermagem responderam ter realizado ou participado de pesquisas no período considerado, 11 informaram que aplicaram os resultados dos estudos na própria instituição $(91,7 \%)$ e 8 explicitaram que tinham conhecimento de que os resultados de seus trabalhos também foram aplicados em outros serviços hospitalares (Tabela 7).

\section{TABELA 7 - HOSPITAIS ESTUDADOS POR TIPO DE ESTABELECIMENTO SEGUNDO A APLICAÇÃO DOS RESULTADOS DAS PESQUISAS - MUNICÍPIO DE SÃO PAULO, ABRIL DE 1994}

\begin{tabular}{cccccc}
\hline & \multicolumn{5}{c}{ Aplicação das Pesquisas } \\
\cline { 2 - 6 } TIPO & PRÓPRIA INSTITUIÇÃO & \multicolumn{2}{c}{ OUTRAS INSTITUIÇÕES } \\
& 6 & NÃO & SIM & NÃO & NÃO SABE \\
\hline Público & 5 & 1 & 5 & 1 & 1 \\
Privado & 11 & - & 3 & - & 2 \\
\hline & $(91,7 \%)$ & $(8,3 \%)$ & $(66,7 \%)$ & $(8,3 \%)$ & $(25,0 \%)$ \\
\hline
\end{tabular}

Os respondentes dos hospitais públicos esclareceram, que os estudos realizados nestes hospitais contribuíram para: intensificar precauções universais no controle das infecções hospitalares; modificar o conteúdo programático de um curso; introduzir uma nova técnica de curativo; modificar assistência prestada uma vez que a maioria dos estudos realizados emergiram de problemas identificados no dia-a-dia de trabalho. Quanto os hospitais privados, as enfermeiras que responderam essa questão, informaram que algumas pesquisas realizadas produziram a modificação da prática de enfermagem e conseqüente melhoria na qualidade do atendimento prestado ao paciente.

Os dados apresentados indicam que a incorporação dos resultados de pesquisas já se faz presente nas instituições estudadas, principalmente quando o objeto de estudo emerge de problemas percebidos no processo de assistir o paciente hospitalizado. 
$\mathrm{Da}$ análise dos dados depreende-se que estudos voltados para o controle das infecções hospitalares, implantação do instrumento de sistematização da assistência de enfermagem em unidade de terapia intensiva e na consulta de enfermagem, técnicas de curativo e de suporte nutricional estavam sendo utilizados em outras instituições.

Foi informado também, que serviços de enfermagem receberam várias solicitações de enfermeiras por meio de consulta pessoal, telefônica e escrita, com vistas a obtenção de maiores esclarecimentos quer sobre os trabalhos publicados quer quanto a dúvidas sobre aplicação de determinada técnica Outra forma apontada foi a realização de visita "in loco" para observação da implantação do instrumento e das técnicas supracitadas.

Além desta troca de informações entre os estabelecimentos de saúde uma instituição apontou o recebimento de convites para realizar palestras em outros serviços hospitalares a fim de divulgar os trabalhos realizados.

\section{Facilidades e dificuldades}

A inquietação quanto a existência ou não do estímulo institucional e do serviço de enfermagem para o desenvolvimento de pesquisas, foi sanada em parte, uma vez que nos achados da pesquisa percebeu-se que 12 (92,3\%) respondentes informaram estar implementando ações visando a este fim.

Nos Quadros 1 e 2 estão explicitadas as facilidades e dificuldades listadas pelo serviços de enfermagem no que tange ao desenvolvimento de pesquisas no período considerado, em hospitais dos setores público e privado.



* Preservou-se a linguagem dos respondentes. 


\section{QUADRO 2 - FACILIDADES E DIFICULDADES CITADAS PELOS SERVIÇOS DE ENFERMAGEM QUANTO À REALIZAÇÃO DE PESQUISAS NO PERÍODO DE 1989 A 1993 NOS HOSPITAIS PRIVADOS ESTUDADOS - MUNICÍPIO DE SÃO PAULO,}

ABRIL DE 1994

\begin{tabular}{|c|c|c|}
\hline HOSPITAL & FACILIDADES* & DIFICULDADES \\
\hline A2 & $\begin{array}{l}\text { "Biblioteca e grupos de interesse na } \\
\text { instituição; médicos e enfermeiras de } \\
\text { renome que facilitam o acesso à } \\
\text { congressos; convites de outras instituições } \\
\text { que estimulam o crescimento do grupo; } \\
\text { contato direto com docentes USP e EPM; } \\
\text { concessão de horários para a realização } \\
\text { de trabalho, reuniões, cursos de mestrado } \\
\text { e doutorado". }\end{array}$ & $\begin{array}{l}\text { "Resistência ou acomodação de algumas } \\
\text { enfermeiras; falta de um membro efetivo que } \\
\text { vincule hospital/universidade". }\end{array}$ \\
\hline B2 & $\begin{array}{l}\text { "Liberação do uso de tempo do serviço e } \\
\text { de recursos materiais; trabalhos externos } \\
\text { são facilitados por reposição de horário". }\end{array}$ & \\
\hline $\mathrm{C} 2$ & $\begin{array}{l}\text { "Recursos oferecidos, maleabilidade de } \\
\text { horário". }\end{array}$ & "Até pouco tempo nós tínhamos horário fixo". \\
\hline $\mathrm{D} 2$ & & "Falta de um programa para esse fim". \\
\hline E2 & "Disponibilidade de recursos materiais". & $\begin{array}{l}\text { "A instituição não libera tempo do horário de } \\
\text { trabalho da enfermeira para a pesquisa". }\end{array}$ \\
\hline G2 & & $\begin{array}{l}\text { "Empresa privada (pacientes particulares), } \\
\text { corpo clínico aberto e diversificado que } \\
\text { dificultam o desenvolvimento da pesquisa". }\end{array}$ \\
\hline
\end{tabular}

* Preservou-se a linguagem dos respondentes.

** O hospital F2 não respondeu a questão.

Nos estabelecimentos públicos as facilidades mais expressivas foram: estímulo institucional e apoio do serviço de enfermagem, criação de um centro de estudos e integração serviço/escola; liberação das enfermeiras para a realização de cursos e freqüência a eventos; apoio de enfermeiras preparadas da própria instituição e de professores contratados; disponibilidade de campo para a realização de pesquisas. Quanto às dificuldades citadas, emergiram entre outras, a falta de conhecimento de metodologia de pesquisa; a necessidade de mudar o comportamento da enfermeira assistencial face a assunção das atividades de pesquisa e carência de recursos financeiros e materiais destinados à produção e divulgação dos estudos realizados. No Hospital $G_{1}$, no qual as enfermeiras participaram apenas de uma pesquisa conduzida por grupo multiprofissional percebeu-se uma contradição nas informações prestadas, pois apontaram como facilidade a disponibilidade de pessoal e como dificuldade o "número de profissionais". Os dados da Tabela 3 mostram que nesse estabelecimento a relação enfermeira por 10 leitos é 5,4 e a de pessoal de enfermagem é de 28 por 10 leitos, o que permite inferir que nesta instituição o número de enfermeiras e o de pessoal de enfermagem, como um todo, não deveriam constituir dificuldade e sim facilidade, quando comparado com os outros hospitais estudados. 
Nos hospitais privados, as facilidades mais citadas dizem respeito à disponibilidade de recursos materiais e de tempo; apenas uma instituição informou dispor de outros recursos como biblioteca e grupos de interesse, além de interação estabelecida com docentes de duas escolas de enfermagem e da oportunidade de enfermeiras realizarem cursos de pós-graduação "sensu stricto". Em relação às dificuldades, emergiram a falta de liberação de horário ou horário não fixo de trabalho; resistência ou acomodação de algumas enfermeiras; falta de articulação entre o hospital e a universidade e de programa visando o desenvolvimento de pesquisa (Quadro 2).

Ressalta-se neste estudo a relevância de algumas estratégias adotadas, tais como: apoio proporcionado por docentes de duas escolas de enfermagem, membros da CEPEn/SP e/ou enfermeiras pesquisadoras contratadas com vistas a orientar os trabalhos de pequisa; estímulo para a freqüência a cursos de metodologia de pesquisa e de pós-graduação "sensu stricto" e criação de centros de pesquisas em enfermagem. Salienta-se ainda que um hospital manifestou a exigência de produção científica anual das enfermeiras pertencentes à instituição, além das estratégias já referidas.

As respostas obtidas, não nos permite afirmar se existe ou não uma política de recursos humanos institucional que possa nortear o desenvolvimento das enfermeiras assistenciais quanto à obtenção de títulos acadêmicos e paralelamente o correspondentes plano de ascensão na carreira profissional. Esta é uma questão que tem nos preocupado e um dos objetos da continuação deste estudo.

\section{CONCLUSÕES}

Dos resultados do estudo extraíram-se as seguintes conclusões:

- houve homogeneidade na distribuição percentual do pessoal de enfermagem atuante nos serviços pesquisados. Ocorreu aumento do número de enfermeiras e auxiliares de enfermagem e redução acentuada do contingente de atendentes de enfermagem o que parece indicar que os hospitais estudados estão contando com pessoal mais qualificado em decorrência da aplicação da legislação do Exercício Profissional de Enfermagem;

- no período de 1989 a 1993, os respondentes informaram que foram produzidas 114 pesquisas nos hospitais públicos e 40 nos privados. O número de estudos publicados foi menor do que os apresentados em eventos científicos;

- há indicativos de que tem ocorrido a incorporação dos resultados destas pesquisas quer nas instituições estudadas quer em outras, principalmente quando o objeto de estudo emerge de problemas percebidos no processo de assistir o paciente hospitalizado;

- as facilidades mais expressivas para o desenvolvimento de pesquisa nas instituições públicas foram o apoio institucional e do serviço de enfermagem; criação de 
centros de estudos e integração serviço/escola; liberação para freqüência a cursos e eventos científicos; apoio de enfermeiras preparadas da própria instituição e de professores contratados e disponibilidade de campo para a realização de investigações. Nos estabelecimentos privados as facilidades mais citadas foram a disponibilidade de tempo e de recursos materiais; freqüência a eventos científicos e cursos de aprimoramento;

- quanto às dificuldades sentidas emergiram nos estabelecimentos públicos a falta de conhecimento, por parte das enfermeiras, de metodologia de pesquisa; a carência de recursos financeiros e materiais destinados à produção e divulgação dos estudos realizados; e a necessidade de mudança de comportamento da enfermeira assistencial face a assunção das atividades de pesquisas. Nos privados as mais citadas foram a falta de liberação de horário; resistência ou acomodação de algumas enfermeiras; falta de articulação entre o serviço e a universidade e de um programa com vistas ao desenvolvimento de pesquisas.

\section{NURSING RESEARCH SITUATION IN HOSPITAL IN THE CITY OF SÃO PAULO}

The objective of this study is to show nursing research situation in public and private hospitals in the city of São Paulo, form 1989 to 1993. It is an exploratory and descriptive research. The questionnaire was answered by nursing directors of 14 hospitals -7 public and private. 114 researches were developed in public hospitals and 40 in private hospitals. The results demonstrated that the majority of studies were developed by four or more authors and most of them were presented in scientific events. The facilities and difficulties to develop research and to implement the results in nursing practice were also pointed out. Research centers, faculty-practice nursing, and admission of nurses researcher were registered ad strategies to develop research in some of these institutions.

UNITERMOS: research, nursing research, scientific production

\section{SITUACIÓN DE LA INVESTIGACIÓN EN ENFERMERÍA EN HOSPITALES DE SÃO PAULO - BRASIL}

El propósito del presente estudio es la descripción de la situación de la investigación en enfermería en los hospitales de la ciudad de São Paulo, Brasil, en el período de 1989 hasta 1993. El diseño utilizado fue exploratoriodescriptivo, abarcando 14 instituciones hospitalarias, de las cuales 7 corresponden al sector público y 7 al sector privado. En los primeros fueron realizadas 114 investigaciones y 40 en los hospitales del sector privado. 
Hubo predominancia de investigaciones presentadas en coloquios científicos. Las facilidades y las dificultades para la utilización de sus resultados en la atención de enfermería, en especial cuando el objeto del estudio tuvo su origen en el proceso asistencial. Para el desarrollo de la investigación en las instituciones estudiadas, fueron empleadas algunas estrategias entre ellas: centros de investigaciones, integración servicio-universidad y contratación de enfermeras investigadoras.

UNITERMOS: investigación, investigación en enfermería; producción científica.

\section{REFERÊNCIAS BIBLIOGRÁFICAS}

01. BRASIL. Ministério da Saúde. Secretaria Nacional das Ações Básicas de Saúde. Divisão Nacional de Organização de Serviços de Saúde. Terminologia básica em saúde. Brasília, Centro de documentação, 1983. (Série B: textos básicos de saúde n. 4).

02. CIANCIARULLO, T.I.; SALZANO, S.D.T. A enfermagem e a pesquisa no Brasil. Rev.Esc.Enf.USP, v. 25, n. 2, p. 195-215, 1991.

03. CONSELHO FEDERAL DE ENFERMAGEM. Exercício de enfermagem nas instituições de saúde do Brasil 1982/1983: força de trabalho em enfermagem, Rio de Janeiro, 1985, v. 1.

04. CHASKA, N. L. The nursing profession: a time to speak. New York, McGraw-Hill, 1983.

05. HOSRLEY, J. A. Using research to import nursing practice: a guide. CURN project. Philadelphia, Saunders, 1983.

06. JOLLEY, M.; ALLAN, P. Current issues in nursing. London, Chapman and Hall, 1989.

07. MOLONEY, M. M. Professionalization of nursing: current issues and trends Philadelphia, Lippincott, 1986.

08. NOGUEIRA, M.J. de C. A pesquisa em enfermagem no Brasil: retrospectiva histórica. Rev. Esc. Enf. USP, v. 16, n.1, p. 17-26, 1982.

09.

Pesquisa em enfermagem comunitária, In: SEMINÁRIO NACIONAL DE PESQUISA EM ENFERMAGEM, 4, São Paulo, 1985. Anais. São Paulo, ABEn, 1985. p. 45-59.

10.PHILLIPS, L.R.F. A clinician's guide to the critique and utilization of nursing research. Norwalk, Appleton-Century-Crofts, 1986.

11. STEFANELLI, M. C.; FRIENDLANDER, M.R. Importância dos programas de pósgraduação para o desenvolvimento técnico-científico da enfermagem. /Reunião: desenvolvimento da enfermagem para a América Latina. Rio de Janeiro, 1992. 


\section{ANEXO I}

Cara colega:

Os membros da comissão de Estudos de Pesquisa em Enfermagem da ABEnSP, entre outras atividades, tem ministrado cursos de iniciação à pesquisa para enfermeiras do Estado de São Paulo.

Visando o aprimoramento destes cursos achamos oportuno realizar este estudo para descrevermos a situação da pesquisa em enfermagem, em hospitais do município de São Paulo.

A sua colaboração é imprescindível, para podermos desenvolver o estudo.

Gostaríamos de reforçar que neste momento, estamos tão somente procurando identificar pesquisas e não trabalhos científicos em geral.

Certas de contarmos com a sua participação, aguardamos resposta até 12.04.94.

Obrigada pela atenção

Coordenadora da CEPEn-SP 
1. Nome da Instituição

2. Endereço

CEP

Município

3. Vinculação administrativa:

setor público: federal ( ) estadual ( ) municipal ( )

setor privado: ( )

4. Tipo de hospital:

- geral ( )

. de especialidade ( )

. especializado ( )

5. Finalidade

. Assistência ( )

. Ensino ( )

. Pesquisa ( )

6. $\quad N^{\circ}$ de leitos disponíveis: desativados:

7. Quadro de pessoal de enfermagem: citar $0 \mathrm{n}^{\circ}$ disponível de cada categoria funcional.

. enfermeira técnico de enfermagem

. auxiliar de enfermagem atendente de enfermagem

8. Nos últimos cinco anos (1989 a 1993) as enfermeiras dessa instituição desenvolveram pesquisas na área de saúde?

Não ( ) Explicar as razões

$$
\begin{aligned}
& \text { Sim ( ) Desenvolvidas somente por enfermeiras ( ) } \\
& \text { Desenvolvidas por grupo multiprofissional ( ) }
\end{aligned}
$$


Neste caso preencher o quando abaixo:

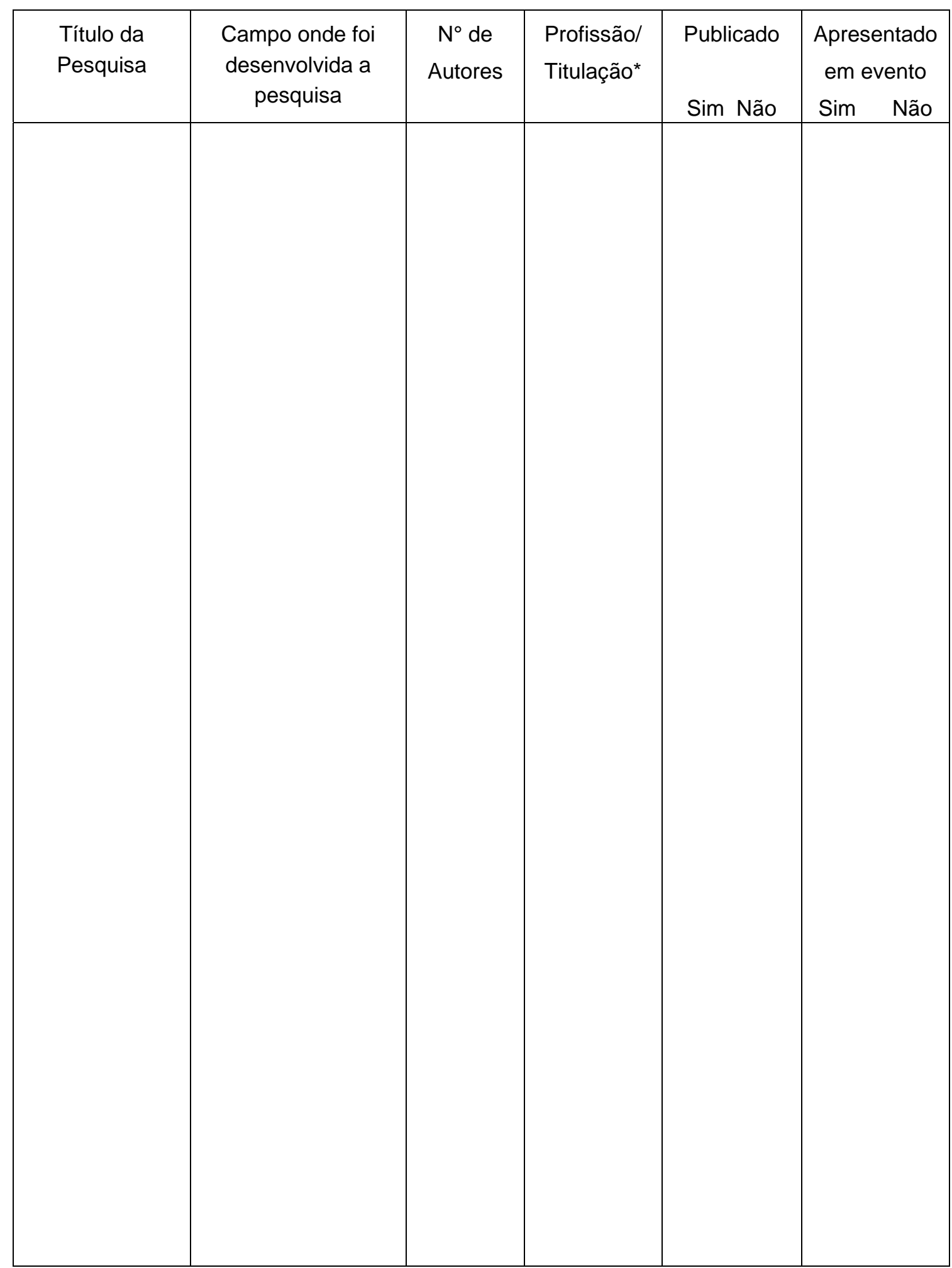

*Profissão: Enfermeiro, Psicólogo, Médico, etc.

Se estudante usar um círculo: $\quad G \quad$ E $\quad M \quad$ D

$\mathrm{G}=$ Graduação $\quad \mathrm{E}=$ Especialização

$M=$ Mestrado $\quad D=$ Doutorado 
9. Os resultados dos estudos realizados e citados no item 8 foram ou estão sendo aplicados na Instituição?

$\operatorname{Sim}($ )

Como?

Não ( ) Justificar as razões

10. A Instituição e o serviço de enfermagem têm estimulado o desenvolvimento da pesquisa institucional?

Sim ( ) Como?

Não ( ) Discriminar as razões

11. Você tem conhecimento se os resultados de pesquisas desenvolvidas por enfermeiras de sua instituição estão sendo utilizados em outras instituições?

Sim ( ) Como?

Não ( ) Não Sei ( )

12. Quais as facilidades e dificuldades para o desenvolvimento da pesquisa pelas enfermeiras nessa Instituição? 